\title{
Real-World Outcomes of Adjuvant Chemotherapy for Node-Negative and Node-Positive HER2-Positive Breast Cancer
}

\author{
Zachary Veitch, MDª, Omar F. Khan, MD'b; Derek Tilley, MScc; Domen Ribnikar, MDa; \\ Xanthoula Kostaras, MSc'; Karen King, MDd; Patricia Tang, MD ${ }^{\text {b }}$ and Sasha Lupichuk, MD, MSc ${ }^{b}$
}

\begin{abstract}
Background: Comparative real-world outcomes for patients with HER2-positive (HER2+) breast cancer receiving adjuvant trastuzumab outside of clinical trials are lacking. This study sought to retrospectively characterize outcomes for patients with node-negative and node-positive breast cancer receiving adjuvant trastuzumab in combination with docetaxel/cyclophosphamide (DCH), docetaxel/carboplatin/trastuzumab (TCH), or fluorouracil/epirubicin/cyclophosphamide followed by docetaxel/trastuzumab (FECDH) chemotherapy in Alberta, Canada, from 2007 through 2014. Methods: Disease-free survival and overall survival (OS) analyses for node-negative cohorts receiving DCH $(n=111)$ or TCH $(n=371)$ and node-positive cohorts receiving FEC-DH $(n=146)$ or TCH $(n=315)$ were compared using chi-square, Kaplan-Meier, or Cox multivariable analysis where appropriate. Results: Median follow-up was similar in node-negative (63.9 months) and node-positive (69.0 months) cohorts. The 5-year OS rates in patients with node-negative disease receiving $\mathrm{DCH}$ or $\mathrm{TCH}$ were similar $(95.2 \%$ vs $96.9 \%$; $P=.268$ ), whereas 5-year OS rates were higher but nonsignificant for patients with node-positive disease treated with FEC-DH compared with $\mathrm{TCH}$ (95.2\% vs $91.4 \% ; P=.160)$. Subgroup analysis of node-positive cohorts showed significantly improved OS with FEC-DH versus TCH in patients with estrogen receptor (ER)/ progesterone receptor (PR)-positive breast cancer (98.3\% vs $91.6 \%$, respectively; $P=.014)$. Conversely, patients with ER/PR-negative disease showed a nonsignificant trend toward higher OS rates with TCH versus FEC-DH (91.6\% vs $83.3 \%$, respectively; $P=.298$ ). Given the retrospective design, we were unable to capture all potential covariates that may have impacted treatment assignment and/ or outcomes. Furthermore, cardiac toxicity data were unavailable. Conclusions: Survival rates of patients with HER2+ breast cancer in our study are comparable to those seen in clinical trials. Our findings support chemotherapy de-escalation in patients with node-negative disease and validate the efficacy of FEC-DH in those with nodepositive disease.
\end{abstract}

J Natl Compr Canc Netw 2019;17(1):47-56 doi: $10.6004 /$ jnccn.2018.7066

\footnotetext{
aPrincess Margaret Cancer Centre, Toronto, Ontario; ${ }^{b}$ Tom Baker Cancer Centre, Calgary, Alberta; 'CancerControl Alberta, Alberta Health Services, Calgary, Alberta; and d Cross Cancer Institute, Edmonton, Alberta, Canada.
}

Amplification or overexpression of the HER2 gene comprises $15 \%$ to $25 \%$ of early-stage breast cancers. ${ }^{1,2}$ Associated with an aggressive phenotype, survival for HER2-positive (HER2+) breast cancer has significantly improved with the use of HER2-targeted therapies, such as trastuzumab. ${ }^{3-5}$ However, outcomes for patients with HER2+ breast cancer treated outside of clinical trials are limited. ${ }^{6,7}$

In small, node-negative, HER2+ tumors, the benefit of adding trastuzumab to adjuvant chemotherapy is well described. ${ }^{8-11}$ However, the potential for overtreatment and toxicity associated with use of third-generation chemotherapy regimens is of concern. After the E1199 clinical trial ${ }^{12}$ established the equivalence of docetaxel every 3 weeks and weekly paclitaxel, 2 single-arm phase II trials evaluated the utility of short-course docetaxel/cyclophosphamide (DCH for 4 cycles) ${ }^{13}$ or paclitaxel (weekly for 12 weeks) ${ }^{14}$ in combination with trastuzumab for lowrisk HER2+ breast cancer. Both trials demonstrated high disease-free survival (DFS) and/or recurrence-free survival rates $(>97 \%)$.

In node-positive HER2+ disease, the benefits of adjuvant trastuzumab in combination with chemotherapy have been shown in the BCIRG- $006^{5}$ and NSABP B-31/ NCCTG N9831 ${ }^{4}$ clinical trials. Treatment with chemotherapy plus trastuzumab was superior to chemotherapy alone. In the final update of BCIRG-006, AC-TH (doxorubicin/cyclophosphamide followed by docetaxel/ trastuzumab) was associated with nonsignificantly higher overall survival (OS) compared with TCH (docetaxel/ carboplatin/trastuzumab) at the detriment of more chemotherapy and higher rates of cardiac and leukemic events. ${ }^{15}$ Despite an abundance of data comparing AC followed by taxane/trastuzumab to TCH, a paucity of data exists comparing FEC-DH (fluorouracil/epirubicin/cyclophosphamide followed by docetaxel/trastuzumab) versus either TCH or AC followed by taxane/ trastuzumab in the adjuvant setting despite inclusion in ASCO guidelines. ${ }^{16}$

See JNCCN.org for supplemental online content. 
We retrospectively evaluated survival outcomes for 2 groups of patients with HER2+ disease: those with (1) node-negative disease receiving either $\mathrm{DCH}$ or $\mathrm{TCH}$, and (2) node-positive disease receiving either FEC-DH or $\mathrm{TCH}$ in the adjuvant setting.

\section{Methods}

\section{Patient Selection}

All women diagnosed from 2007 through 2014 with stage I-III, HER2+ breast cancer who received DCH for 4 cycles, TCH for 6 cycles, or FEC-DH for 6 cycles followed by up to 1 year of trastuzumab in the province of Alberta, Canada, were identified using the Alberta Cancer Registry (ACR). Patients were stratified into 2 cohorts: those with (1) node-negative disease who received $\mathrm{DCH}$ or TCH or (2) node-positive disease who received FEC-DH or TCH (Figure 1).

Patient and pathology characteristics (Tables 1 and 2 , for node-negative and node-positive cohorts, respectively), treatment characteristics (Tables 3 and 4 , for node-negative and node-positive cohorts, respectively), and recurrence information (date and sites: local, bone, visceral, brain) were collected from the ACR and, when required, manual chart review. Patient characteristics included age, menopausal status, and comorbidity data using the updated Charlson comorbidity index (U-CCI). ${ }^{17}$ Pathology characteristics included AJCC tu- mor size, tumor grade, lymphovascular invasion (LVI), and histologic type. Treatment characteristics included chemotherapy regimen and cycles completed, surgery type, radiotherapy prescription, and adjuvant hormone therapy prescription. This study was approved by the Health Research Ethics Board of Alberta.

\section{Statistics}

Patient characteristics were compared using chi-square test, Fisher exact test, $t$-test, or Mann-Whitney U test where appropriate. For survival calculations, time from breast surgery to last follow-up or event was used. DFS, OS, and breast cancer-specific survival (BCSS) were compared using the Kaplan-Meier method (with log-rank analyses). A Cox proportional hazards model was used for survival estimates in multivariable analysis of treatment and patient characteristics. Hazard ratios (HRs) and 95\% CIs are reported. For all tests, $P<.05$ was considered significant. Statistics were performed using SigmaPlot V13 (Systat Software, Inc) or SPSS Statistics, version 19 (IBM Corporation).

\section{Results}

\section{Patients}

A total of 1,006 patients with HER2+ breast cancer receiving 1 of 3 treatment regimens $(\mathrm{DCH}, \mathrm{TCH}$, or FEC-DH) were identified over a 7 -year period. Overall, 19 patients with node-positive disease received

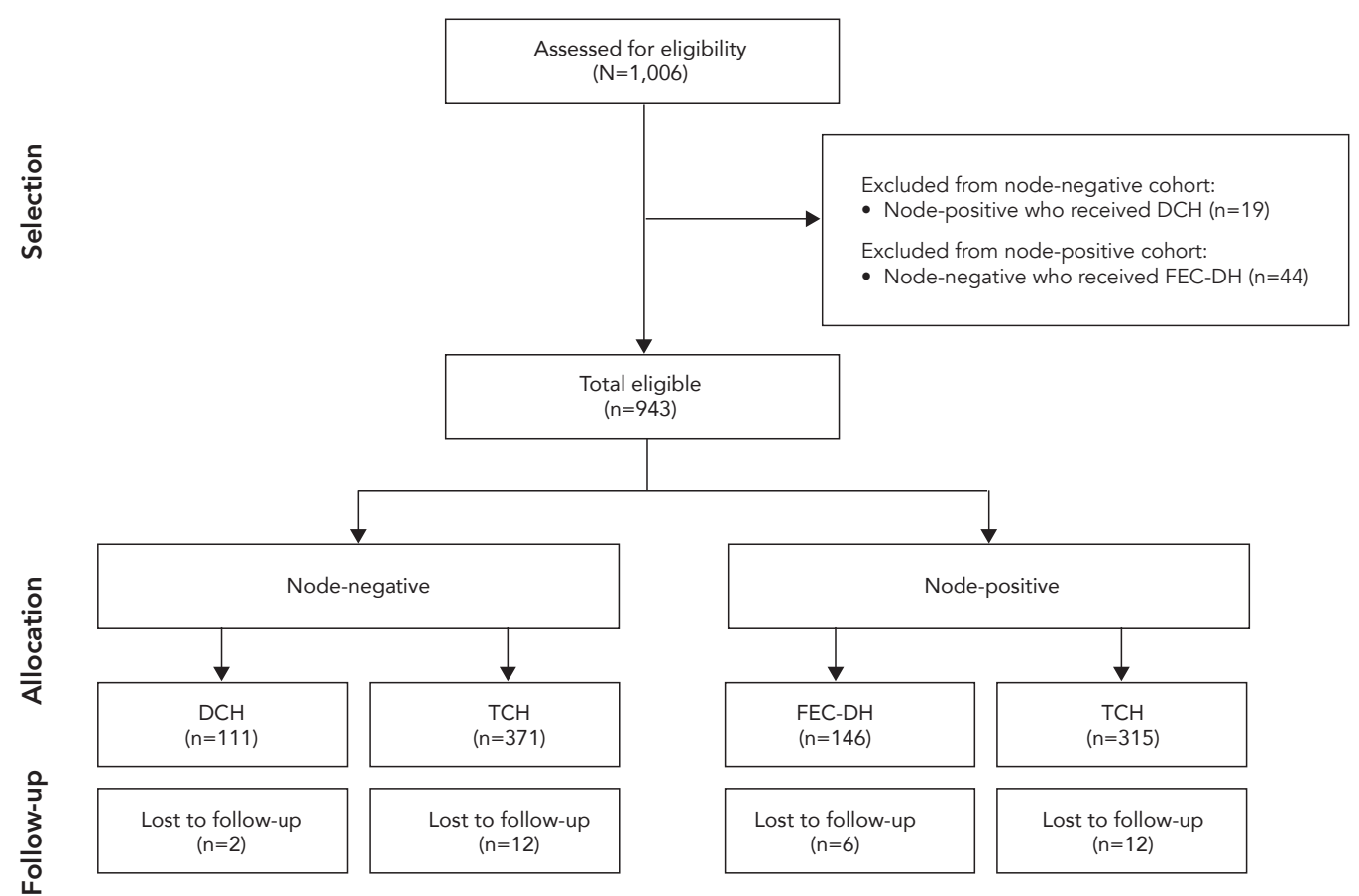

Figure 1. Selection and allocation of node-negative and node-positive cohorts.

Abbreviations: DCH, docetaxel/cyclophosphamide; FEC-DH, fluorouracil/epirubicin/cyclophosphamide followed by docetaxel/trastuzumab; $\mathrm{TCH}$, docetaxel/carboplatin/trastuzumab. 
$\mathrm{DCH}$ and were thus excluded from the node-negative cohort. Conversely, 44 patients with node-negative disease received FEC-DH and were excluded from the node-positive cohort. A total of 943 patients were eligible for allocation to the node-negative (DCH or $\mathrm{TCH}$ ) and node-positive (FEC-DH or TCH) cohorts (Figure 1).

\section{Node-Negative Cohort}

\section{Characteristics}

In the node-negative cohort, 482 patients $(\mathrm{DCH}, \mathrm{n}=111$; $\mathrm{TCH}, \mathrm{n}=371$ ) were included for analysis (Table 1). Clinicopathologic features were not significantly different between DCH and TCH cohorts. Patients receiving DCH were slightly

\begin{tabular}{|c|c|c|c|c|c|}
\hline & \multicolumn{2}{|c|}{$\begin{array}{c}\mathrm{DCH} \\
(\mathrm{N}=111)\end{array}$} & \multicolumn{2}{|c|}{$\begin{array}{c}\mathrm{TCH} \\
(\mathrm{N}=371) \\
\end{array}$} & \multirow[b]{2}{*}{$P$ Value } \\
\hline & $\mathbf{n}$ & $\%$ & $\mathbf{n}$ & $\%$ & \\
\hline Median age (range), y & 56 & (34-74) & 53 & $(28-76)$ & $<.001^{\mathrm{a}}$ \\
\hline Menopausal status & & & & & .467 \\
\hline Premenopausal & 45 & 40.5 & 164 & 44.2 & \\
\hline Postmenopausal & 66 & 59.5 & 207 & 55.8 & \\
\hline $\mathrm{U}-\mathrm{CCl}$ & & & & & .053 \\
\hline 0 & 89 & 80.2 & 317 & 85.4 & \\
\hline 1 & 17 & 15.3 & 50 & 13.5 & \\
\hline$\geq 2$ & 5 & 4.5 & 4 & 1.1 & \\
\hline Histology & & & & & .126 \\
\hline IDC & 94 & 84.7 & 330 & 88.9 & \\
\hline ILC & 6 & 5.4 & 7 & 1.9 & \\
\hline Other & 11 & 9.9 & 34 & 9.2 & \\
\hline T stage & & & & & $.028^{*}$ \\
\hline T1 & 77 & 69.4 & 207 & 55.8 & \\
\hline $\mathrm{T} 2$ & 34 & 30.6 & 160 & 43.1 & \\
\hline $\mathrm{T} 3$ & 0 & 0 & 4 & 1.1 & \\
\hline Unknown & 0 & 0 & 0 & 0 & \\
\hline $\mathrm{N}$ stage & & & & & NA \\
\hline No & 111 & 100 & 371 & 100 & \\
\hline AJCC stage & & & & & $.024^{\mathrm{a}}$ \\
\hline 1 & 77 & 69.4 & 204 & 55.0 & \\
\hline$\|$ & 34 & 30.6 & 166 & 44.7 & \\
\hline III & 0 & 0 & 1 & 0.3 & \\
\hline Tumor grade & & & & & .258 \\
\hline 1 & 2 & 1.8 & 5 & 1.3 & \\
\hline 2 & 35 & 31.5 & 89 & 24.0 & \\
\hline 3 & 74 & 66.7 & 276 & 74.4 & \\
\hline Unknown & 0 & 0 & 1 & 0.3 & \\
\hline LVI & & & & & .102 \\
\hline Present & 25 & 22.5 & 113 & 30.5 & \\
\hline Absent & 84 & 75.7 & 251 & 67.7 & \\
\hline Unknown & 2 & 1.8 & 7 & 1.9 & \\
\hline Hormone receptor status & & & & & .289 \\
\hline$\underline{E R+/ P R+}$ & 90 & 81.1 & 283 & 76.3 & \\
\hline ER-/PR- & 21 & 18.9 & 88 & 23.7 & \\
\hline
\end{tabular}

Abbreviations: DCH, docetaxel/cyclophosphamide; ER, estrogen receptor; IDC, invasive ductal carcinoma; ILC, invasive lobular carcinoma; LVI, lymphovascular invasion; NA, not applicable; PR, progesterone receptor; TCH, docetaxel/carboplatin/trastuzumab; U-CCl, updated Charlson comorbidity index. ${ }^{a} P<.05$. 


\begin{tabular}{|c|c|c|c|c|c|}
\hline & \multicolumn{2}{|c|}{$\begin{array}{l}\text { FEC-DH } \\
(\mathrm{N}=146)\end{array}$} & \multicolumn{2}{|c|}{$\begin{array}{c}\mathrm{TCH} \\
(\mathrm{N}=315) \\
\end{array}$} & \multirow[b]{2}{*}{$P$ Value } \\
\hline & $\mathbf{n}$ & $\%$ & $\mathbf{n}$ & $\%$ & \\
\hline Median age (range), y & 50 & (25-74) & 54 & $(24-81)$ & $.002^{\mathrm{a}}$ \\
\hline Menopausal status & & & & & $.003^{\mathrm{a}}$ \\
\hline Premenopausal & 85 & 58.2 & 137 & 43.5 & \\
\hline Postmenopausal & 61 & 41.8 & 178 & 56.5 & \\
\hline $\mathrm{U}-\mathrm{CCl}$ & & & & & .451 \\
\hline 0 & 120 & 82.2 & 273 & 86.7 & \\
\hline 1 & 23 & 15.8 & 37 & 11.7 & \\
\hline$\geq 2$ & 3 & 2.1 & 5 & 1.6 & \\
\hline Histology & & & & & $.036^{\mathrm{a}}$ \\
\hline IDC & 123 & 84.2 & 288 & 91.4 & \\
\hline ILC & 4 & 2.7 & 2 & 0.6 & \\
\hline Other & 19 & 13.0 & 25 & 7.9 & \\
\hline T stage & & & & & $.002^{\mathrm{a}}$ \\
\hline $\mathrm{T} 1$ & 34 & 23.3 & 110 & 34.9 & \\
\hline $\mathrm{T} 2$ & 87 & 59.6 & 180 & 57.1 & \\
\hline $\mathrm{T3}$ & 24 & 16.4 & 24 & 7.6 & \\
\hline Unknown & 1 & 0.7 & 1 & 0.3 & \\
\hline $\mathrm{N}$ stage & & & & & .324 \\
\hline $\mathrm{N} 1$ & 81 & 55.5 & 195 & 61.9 & \\
\hline $\mathrm{N} 2$ & 43 & 29.5 & 73 & 23.2 & \\
\hline N3 & 22 & 15.1 & 47 & 14.9 & \\
\hline AJCC stage & & & & & $.03^{a}$ \\
\hline I & 0 & 0 & 0 & 0 & \\
\hline$\|$ & 71 & 48.6 & 187 & 59.4 & \\
\hline III & 75 & 51.4 & 128 & 40.6 & \\
\hline Tumor grade & & & & & .280 \\
\hline 1 & 0 & 0 & 5 & 1.6 & \\
\hline 2 & 31 & 21.2 & 60 & 19.2 & \\
\hline 3 & 115 & 78.8 & 248 & 79.2 & \\
\hline Unknown & 0 & & 2 & & \\
\hline LVI & & & & & .097 \\
\hline Present & 109 & 74.7 & 211 & 67.0 & \\
\hline Absent & 35 & 24.0 & 99 & 31.4 & \\
\hline Unknown & 2 & 1.3 & 5 & 1.6 & \\
\hline Hormone receptor status & & & & & .407 \\
\hline $\mathrm{ER}+/ \mathrm{PR}+$ & 115 & 78.8 & 237 & 75.2 & \\
\hline ER-/PR- & 31 & 21.2 & 78 & 24.8 & \\
\hline
\end{tabular}

Abbreviations: ER, estrogen receptor; FEC-DH, fluorouracil/epirubicin/cyclophosphamide followed by docetaxel/trastuzumab; IDC, invasive ductal carcinoma; ILC, invasive lobular carcinoma; LVI, lymphovascular invasion; PR, progesterone receptor; TCH, docetaxel/carboplatin/trastuzumab; U-CCl, updated Charlson comorbidity index. ${ }^{a} P<.05$.

older (median age, 56 years; range, 34-74 years) compared with those receiving TCH (median age, 53 years; range, 2876 years; $P<.001$ ). A trend toward lower (U-CCI, 0) patient comorbidities was seen with TCH (85.4\%) relative to DCH (80.2\%; $P=.053)$, and there was an imbalance in the distri- bution of $\mathrm{T} 1$ (more frequently treated with $\mathrm{DCH}$ ) and T2 tumors (more frequently treated with TCH; $P=.028$ ); few T3 tumors were seen in either the $\mathrm{DCH}$ or TCH groups. No significant differences were seen in sequential treatment modalities or completion of chemotherapy (Table 3 ). 
Patterns of relapse are shown in Figure 2. No bone only or brain relapses were seen in the DCH cohort. In the node-negative TCH cohort, brain (evaluated separately) was involved in $32 \%$ of relapses. Relapse characteristics for both cohorts are listed in supplemental eAppendix 1, available with this article at JNCCN.org.

\section{Outcomes}

Median follow-up for the node-negative cohort was 63.9 months. Five-year survival rates remained high for both the DCH and TCH cohorts, with no significant differences seen in DFS (92.3\% vs $95.2 \%$; $P=.485)$, OS (95.2\% vs $96.9 \%$; $P=.268)$, or BCSS $(97.1 \%$ vs $98.3 \%$; $P=.285)$ (Figure 3$)$. In multivariable analysis for OS (Table 5), characteristics with HR $<0.67$ or $>1.5$ and nonsignificant trends toward worse outcomes were seen for DCH relative to TCH (HR, 1.956; $P=.196$ ), T2 relative to T1 tumors (HR, 1.887; $P=.192$ ), premenopausal relative to postmenopausal status (HR, 0.392; $P=.105$ ), and hormone receptor-negative relative to hormone receptor-positive tumors (HR, 2.100; $P=.137$ ). Receipt of radiotherapy, tumor grade, and LVI were not shown to affect survival. Higher number of comorbidities $(\geq 1)$ significantly affected DFS (HR, 2.83; $P=.014)$ but did not affect OS $(P=.932)$ or BCSS $(P=.415)$ in multivariate analysis.

\section{Node-Positive Cohort}

\section{Characteristics}

In the node-positive cohort, 461 patients (FEC-DH, $\mathrm{n}=146$; TCH, $\mathrm{n}=315$ ) were included for analysis (Table 2). Patients receiving FEC-DH were younger (median age, 50 years; range, $25-74$ years) than those receiving TCH (median age, 54 years; range, $24-81$ years; $P=.002$ ). Significantly more patients in the FEC-DH cohort were pre- menopausal (58.2\%) than those receiving TCH $43.5 \%$; $P=.003)$. No differences were seen in number of comorbidities $(P=.451)$.

Comparison of clinical pathologic features between cohorts showed that significantly more patients with larger tumors $(>2 \mathrm{~cm})$ were treated with FEC-DH $(76.6 \%)$ relative to TCH (69.4\%; $P=.002)$. Consequently, more AJCC stage III tumors $(P=.03)$ were treated with FEC-DH $(51.4 \%)$ relative to TCH $(40.6 \%)$. Fewer patients treated with FEC-DH had invasive ductal histology (84.2\%) relative to TCH (91.4\%; $P=.036)$. No significant differences were seen in tumor grade, hormone receptor status, or LVI. Cohorts were balanced for those receiving sequential treatment modalities (Table 4). No significant differences were seen in the number of patients completing chemotherapy $(P=.754)$.

Relapse patterns were similar except for the frequency of local recurrences (FEC-DH, $38 \%$ vs TCH, 13\%) as well as combined bone and visceral metastasis (FEC-DH, $23 \%$ vs TCH, $41 \%$ ) (Figure 2). Relapse characteristics for both cohorts are listed in supplemental eAppendix 2.

\section{Outcomes}

Median follow-up for the node-positive cohort was 69.0 months, and 5-year survival (Figure 4) exceeded $88 \%$ for both cohorts, with a nonsignificant trend favoring FEC-DH over TCH for DFS (92.4\% vs $88.5 \% ; P=.280)$, OS (95.2\% vs $91.4 \%$; $P=.160)$, and BCSS $(95.9 \%$ vs $92.4 \%$; $P=.161)$.

In multivariable analysis (Table 5) for OS, characteristics with $\mathrm{HR}<0.67$ or $>1.5$ and nonsignificant trends toward worse outcomes were seen for TCH relative to FEC-DH (HR, 1.90; $P=.117$ ), premenopausal relative to postmenopausal status (HR, 0.608; $P=.154$ ),

\section{Table 3. Treatment Characteristics for Node-Negative Cohorts}

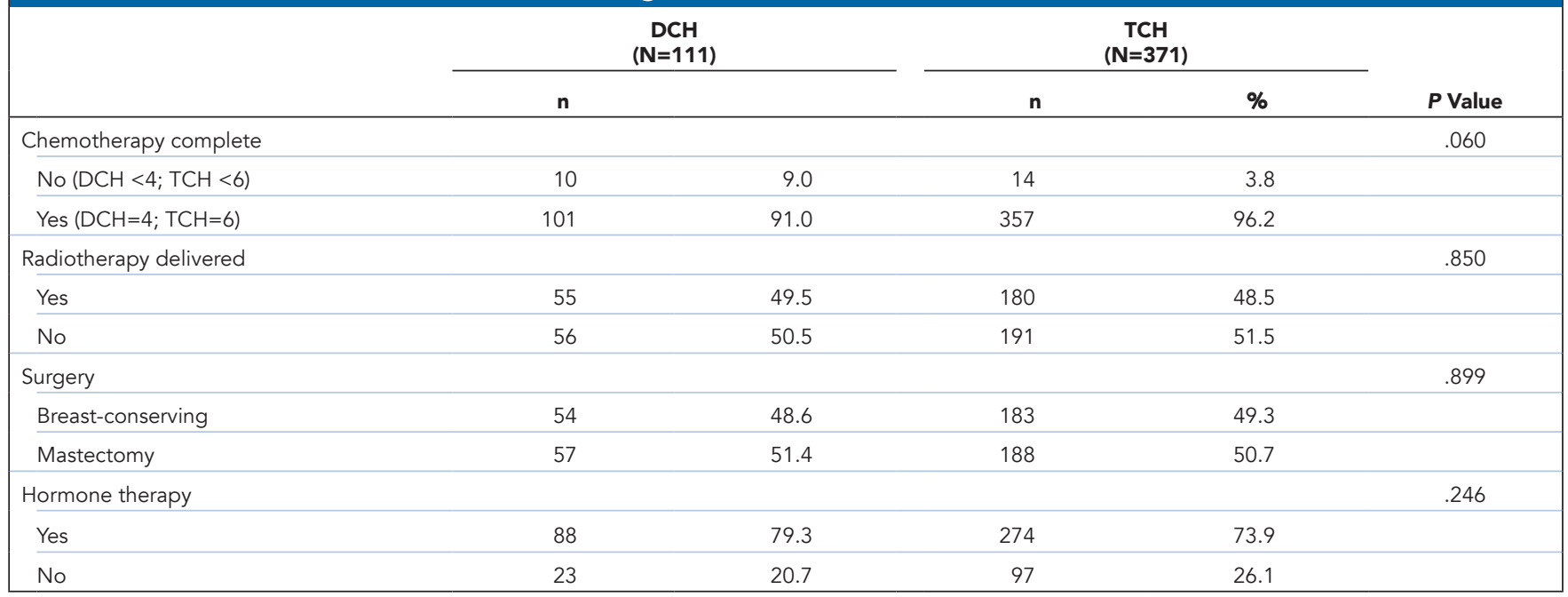

Abbreviations: DCH, docetaxel/cyclophosphamide; TCH, docetaxel/carboplatin/trastuzumab. 


\begin{tabular}{|c|c|c|c|c|c|}
\hline & \multicolumn{2}{|c|}{$\begin{array}{l}\text { FEC-DH } \\
(\mathrm{N}=146)\end{array}$} & \multicolumn{2}{|c|}{$\begin{array}{c}\text { TCH } \\
(\mathrm{N}=315)\end{array}$} & \multirow[b]{2}{*}{$P$ Value } \\
\hline & $\mathbf{n}$ & $\%$ & $\mathbf{n}$ & $\%$ & \\
\hline Chemotherapy complete & & & & & .754 \\
\hline No (FEC-DH <6; TCH <6) & 6 & 4.1 & 15 & 4.8 & \\
\hline Yes (FEC-DH=6; TCH=6) & 140 & 95.9 & 300 & 95.2 & \\
\hline Radiotherapy delivered & & & & & .265 \\
\hline Yes & 129 & 88.4 & 266 & 84.4 & \\
\hline No & 17 & 11.6 & 49 & 15.6 & \\
\hline Surgery & & & & & .247 \\
\hline Breast-conserving & 37 & 25.3 & 97 & 30.8 & \\
\hline Mastectomy & 108 & 74.0 & 218 & 69.2 & \\
\hline Unknown & 1 & 0.7 & 0 & 0 & \\
\hline Hormone therapy & & & & & .470 \\
\hline Yes & 109 & 74.7 & 225 & 71.4 & \\
\hline No & 37 & 25.3 & 90 & 28.6 & \\
\hline
\end{tabular}

Abbreviations: FEC-DH, fluorouracil/epirubicin/cyclophosphamide followed by docetaxel/trastuzumab; TCH, docetaxel/carboplatin/trastuzumab.

grade 3 relative to grade 2 tumors (HR, 1.780; $P=.284$ ), presence relative to absence of LVI (HR, 1.568; $P=.309$ ), and T3 (HR, 2.107; $P=.231$ ) or T2 (HR, 1.925; $P=.163$ ) relative to $\mathrm{T} 1$ tumors.

With respect to OS for nodal status, N3 (HR, 1.948) did not reach statistical significance relative to $\mathrm{N} 1(P=.148)$, but N2 did (HR, 2.314; $P=.032$ ). In analysis for DFS, HRs remained significant for $\mathrm{N} 3$ disease (HR, 3.639; $P=.001$ ) and N2 (HR, 3.037; $P=.002$ ) relative to N1 (supplemental eAppendix 3). This trend continued for BCSS, with N3 (HR, 2.958; $P=.031$ ) and N2 disease (HR, 3.467; $P=.004$ ) showing worse outcomes relative to N1. Lack of radiotherapy ad- versely affected DFS (HR, 3.510; $P=.013$ ) but did not affect OS or BCSS.

\section{Subgroup Analysis}

In subgroup analysis of patients with node-positive hormone receptor-positive tumors (TCH, $\mathrm{n}=238$; FEC-DH, $\mathrm{n}=115)$, OS was superior $(P=.014)$ for those treated with FEC-DH (98.3\%) compared with TCH $(91.6 \% ; P=.018)$ (supplemental eAppendix 4A). DFS was of borderline significance $(P=.056)$, even with balanced nodal status between cohorts. Conversely, subgroup analysis of patients with node-positive hormone receptor-nega-

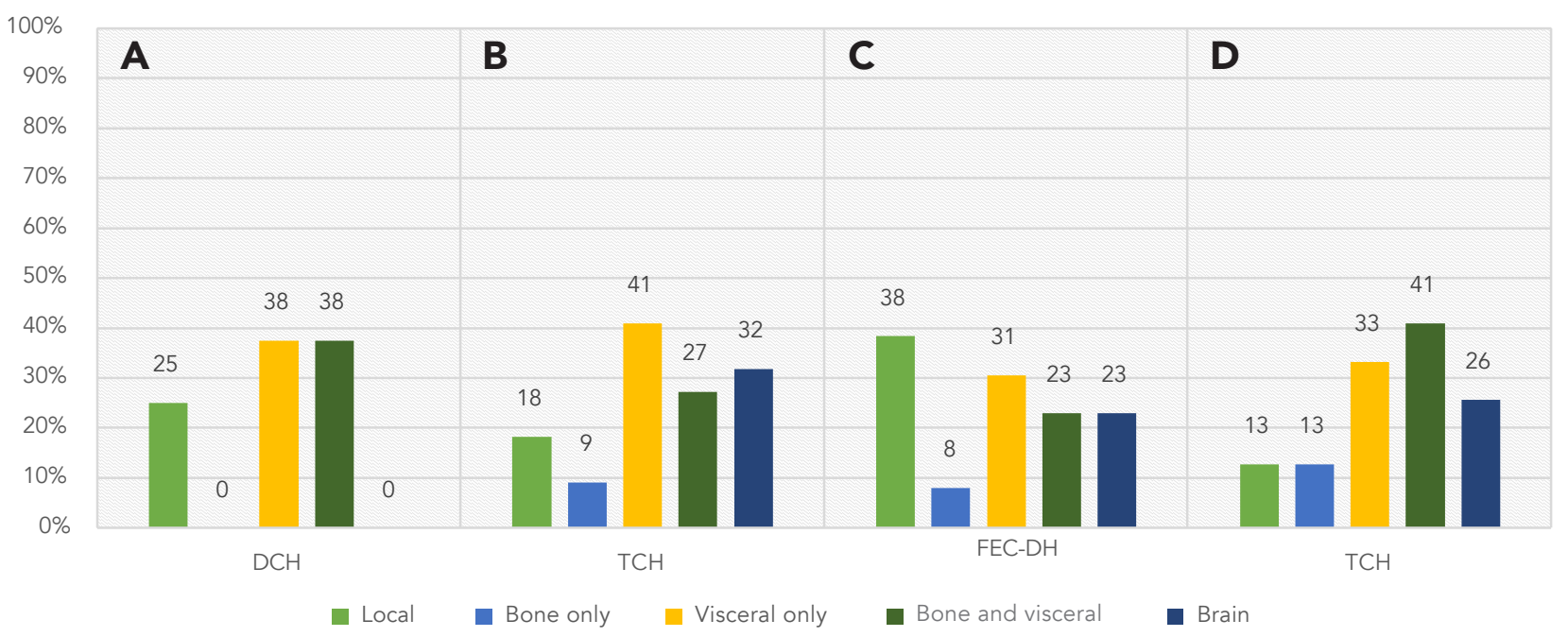

Figure 2. Frequency of recurrence by location and $(A, B)$ node-negative and $(C, D)$ node-positive cohorts for each treatment regimen. Abbreviations: DCH, docetaxel/cyclophosphamide; FEC-DH, fluorouracil/epirubicin/cyclophosphamide followed by docetaxel/trastuzumab; $\mathrm{TCH}$, docetaxel/carboplatin/trastuzumab. 
A

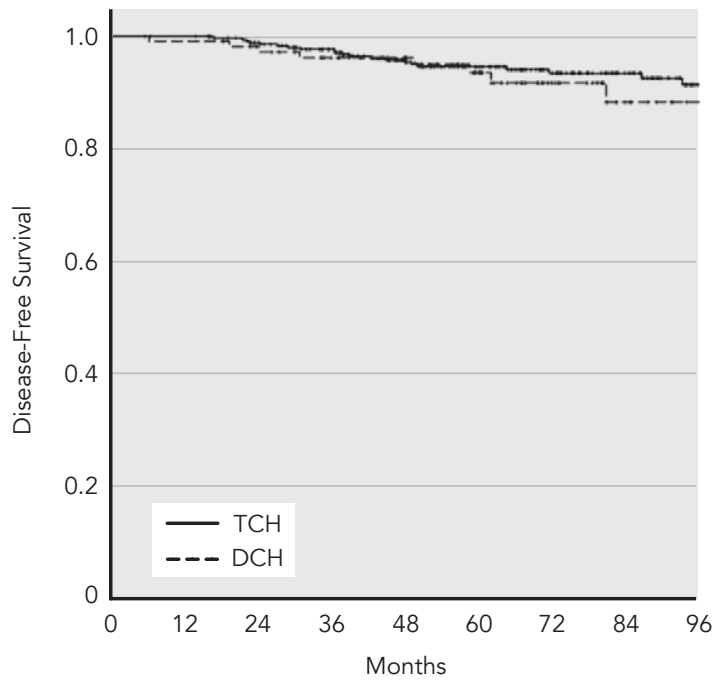

B

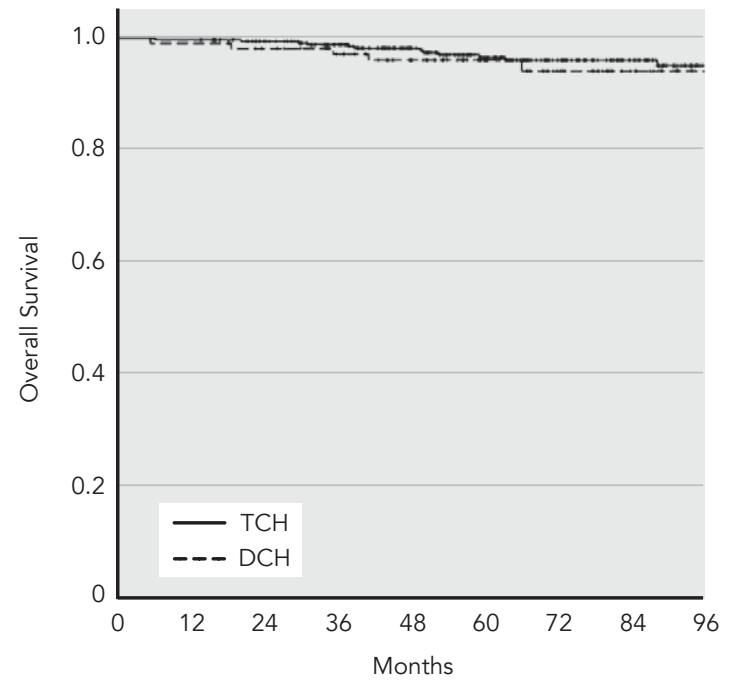

C

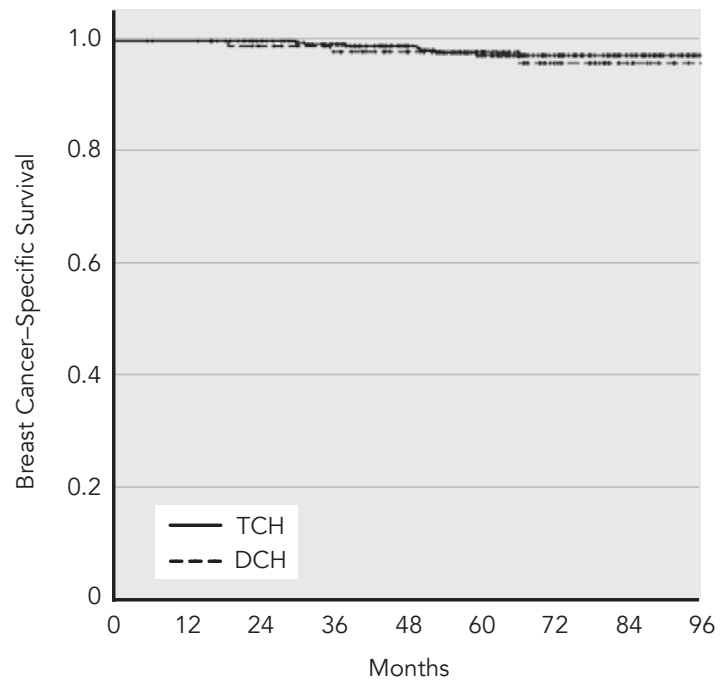

Figure 3. Kaplan-Meier plots for (A) disease-free survival, (B) overall survival, and (C) breast cancer-specific survival for the nodenegative cohort.

Abbreviations: DCH, docetaxel/cyclophosphamide; $\mathrm{TCH}$, docetaxel/carboplatin/trastuzumab. tive tumors showed higher DFS and OS rates for those treated with TCH $(\mathrm{n}=76 ; 88.2 \%$ and $90.8 \%$, respectively) relative to those treated with FEC-DH $(n=30 ; 83.3 \%$ and $83.3 \%$, respectively) ( $P=.272$ and $P=.269$, respectively), although this was nonsignificant (supplemental eAppendix 4B).

\section{Discussion}

This study addresses several areas in which data are limited for different prognostic subgroups of patients with HER2+ disease. Within the node-negative population, OS for DCH and TCH were found to be high (>95\%), despite some imbalance in the number of $\mathrm{T} 1$ and $\mathrm{T} 2$ tumors. Slight discrepancies in age were balanced by equivalence in menopausal status. As a result of improvements in the treatment of HER2+ breast cancer, the landscape for treatment of low-risk HER2+ tumors has shifted toward chemotherapy de-escalation. In a recent single-arm phase II study by Tolaney et al, ${ }^{14}$ single-agent paclitaxel given for 12 weeks in combination with 1 year of adjuvant trastuzumab (APT trial) for small node-negative tumors demonstrated very low recurrence rates with favorable toxicity profiles. Similar results were seen in the single-arm phase II trial using DCH by Jones et al. ${ }^{13}$

Within HER2+, node-positive populations, comparison of FEC-DH with either AC followed by taxane/trastuzumab or TCH in the adjuvant setting has never been performed despite frequent use, phase III evidence, ${ }^{18}$ and inclusion in recent ASCO guidelines. ${ }^{16}$ In the sentinel BCIRG-006 trial, AC-TH compared with TCH was found to be associated with higher DFS ( $80 \%$ vs $78 \%$ ) but not OS in the final 10 -year analysis. ${ }^{15}$ Similarly, for patients with node-positive breast cancer in our study, no significant difference in OS was seen for FEC-DH relative to $\mathrm{TCH}$, yet OS was improved for patients with hormone receptor-positive, HER2+ disease (98.3\% vs 91.6\%).

An evolving body of literature supports biological differences in estrogen receptor-positive (ER+)/HER2+ compared with ER-/HER2+ breast cancer. ${ }^{19,20}$ Coamplification of TOP2A is more common in ER+/HER2+ than ER-/HER2+ disease and could contribute to selective anthracycline benefit. ${ }^{21}$ Furthermore, HER2 may be a less important driver in hormone receptor-positive ${ }^{22}$ breast malignancies, making combination anthracycline/taxane chemotherapy more relevant. Interestingly, when survival was evaluated for hormone receptor-negative tumors, the opposite was true, with $\mathrm{TCH}$ appearing to have higher survival relative to FEC-DH (90.8\% vs $83.3 \%)$ despite lacking statistical power. In the neoadjuvant phase II TRYPHAENA trial, ${ }^{23}$ similar results were seen, with higher pathologic complete response (pCR) rates in ER-/HER2+ tumors treated with $\mathrm{TCH}+$ pertuzumab (84\%) versus FEC-DH + pertuzumab (65\%). Yet, for ER+l HER2+ tumors receiving the same chemotherapy, rates 
A

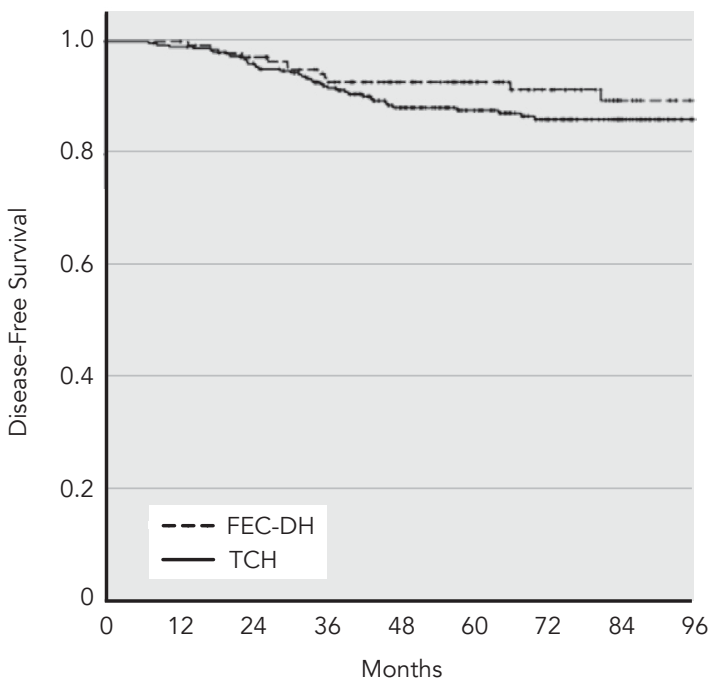

B

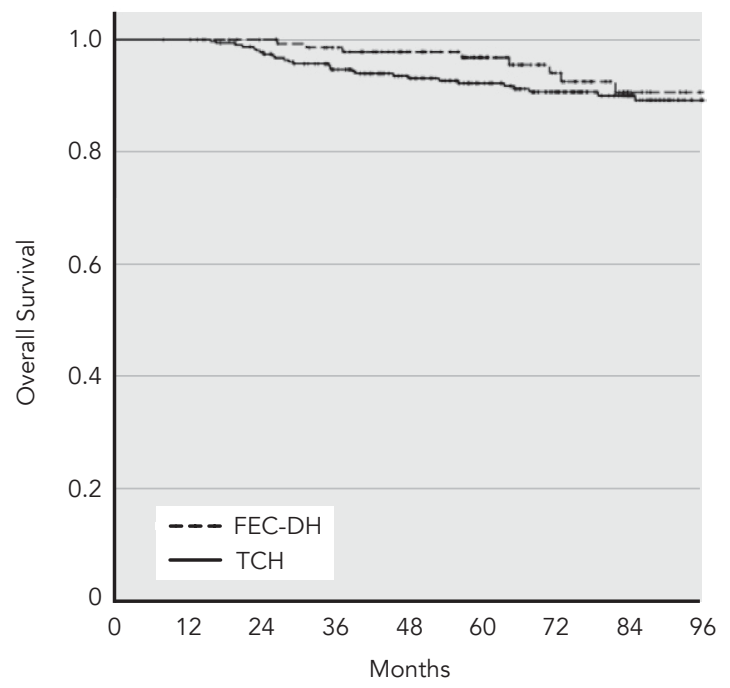

C

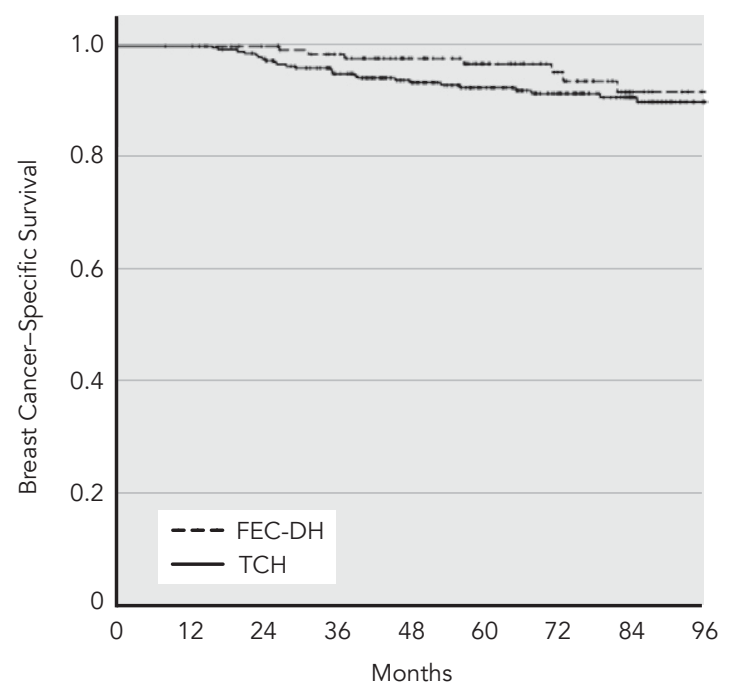

Figure 4. Kaplan-Meier plots for (A) disease-free survival, (B) overall survival, and (C) breast cancer-specific survival for the node-positive cohort. Abbreviations: FEC-DH, fluorouracil/epirubicin/cyclophosphamide followed by docetaxel/trastuzumab; TCH, docetaxel/carboplatin/ trastuzumab. of pCR were similar (50\% vs $49 \%$ ). Patients achieving a total (axillary and breast) pCR had higher 3-year DFS rates than those without a pCR (HR, 0.27). ${ }^{24}$ Similar results have been reported in other neoadjuvant trials. ${ }^{25}$

The addition of other anti-HER2 agents to trastuzumab-based chemotherapy regimens has shown additional benefit in the adjuvant setting. However, these agents are not universally available or accepted without long-term survival data, and therefore the results of this study can still be considered relevant in the current era. In the phase III APHINITY trial, ${ }^{26}$ patients were randomly allocated to receive trastuzumab-based chemotherapy with or without pertuzumab starting with the first cycle of taxane-containing chemotherapy and continuing for up to 18 cycles. Although most patients were treated with an anthracycline-containing regimen (FEC-DH or AC-TH), TCH was permitted. The addition of pertuzumab to standard treatment revealed a statistically significant, albeit small, absolute improvement in invasive DFS (absolute benefit of $1.8 \%$ ) at 3 years. ${ }^{26} \mathrm{In}$ the phase III ExteNET study, patients were randomized to neratinib versus placebo for 1 year following completion of trastuzumab-based adjuvant chemotherapy. Neratinib also yielded a small absolute benefit in invasive DFS events ${ }^{27}$ but with significant grade 3 diarrhea in $40 \%$ of patients. In preplanned subgroup analyses, patients with node-positive or hormone receptor-negative disease derived greater benefit with the addition of pertuzumab. ${ }^{26}$ Conversely, patients with hormone receptor-positive disease derived greater benefit with the addition of neratinib. ${ }^{27}$ Final OS analyses for both APHINITY and ExteNET are awaited. NCCN ${ }^{28}$ and ASCO $^{16}$ guidelines now consider trastuzumab + pertuzumab an option in the adjuvant setting for node-positive disease, and extended anti-HER2 therapy with neratinib in patients with node-positive and hormone receptorpositive disease. Of note, the benefits and toxicities of extended anti-HER2 therapy with neratinib after pertuzumab exposure are unknown.

Given the retrospective design of our study, we were unable to capture all potential covariates that may have impacted treatment assignment and/or outcomes. First, whether performance status or particular comorbidities influenced choice of adjuvant chemotherapy is unknown. We were only able to measure and control for the U-CCI. Within our study, $>95 \%$ of patients had a U-CCI score of 0 to 1 , indicating relatively low levels of comorbidity, and U-CCI interaction was found to be nonsignificant in multivariable testing for OS and BCSS.

Second, we did not measure completion rates of adjuvant trastuzumab. In the ShortHER clinical trial, ${ }^{29} 9$ weeks of adjuvant trastuzumab failed to reach a noninferiority end point compared with 12 months (HR, 1.15, with $95 \%$ CIs crossing the upper boundary of 1.289). In a 
Table 5. Multivariable Analysis of Overall Survival

\begin{tabular}{|c|c|c|c|c|c|c|}
\hline \multirow[b]{2}{*}{ Patient Variables } & \multicolumn{3}{|c|}{ Node-Negative Cohort (A) } & \multicolumn{3}{|c|}{ Node-Positive Cohort (B) } \\
\hline & HR & $95 \% \mathrm{Cl}$ & $P$ Value & HR & $95 \% \mathrm{Cl}$ & $P$ Value \\
\hline \multicolumn{7}{|l|}{ Chemotherapy } \\
\hline $\mathrm{DCH}(\mathrm{A}) / \mathrm{FECDH}(\mathrm{B})$ & 1.956 & $0.707-5.410$ & .196 & 1.900 & $0.851-4.239$ & .117 \\
\hline \multicolumn{7}{|l|}{ Menopausal status } \\
\hline Postmenopausal & \multicolumn{3}{|c|}{ Ref } & \multicolumn{3}{|c|}{ Ref } \\
\hline 0 & \multicolumn{3}{|c|}{ Ref } & \multicolumn{3}{|c|}{ Ref } \\
\hline$\geq 1$ & 0.945 & $0.262-3.415$ & .932 & 0.793 & $0.302-2.078$ & .637 \\
\hline \multicolumn{7}{|l|}{ Tumor grade } \\
\hline 2 & \multicolumn{3}{|c|}{ Ref } & \multicolumn{3}{|c|}{ Ref } \\
\hline 3 & 1.262 & $0.411-3.877$ & .684 & 1.780 & $0.620-5.107$ & .284 \\
\hline \multicolumn{7}{|l|}{ T stage } \\
\hline $\mathrm{T} 1$ & \multicolumn{3}{|c|}{ Ref } & & & \\
\hline $\mathrm{T} 2$ & 1.887 & $0.727-4.897$ & .192 & 1.925 & $0.767-4.831$ & .163 \\
\hline T3 & NA & NA & NA & 2.107 & $0.623-7.123$ & .231 \\
\hline \multicolumn{7}{|l|}{$\mathrm{N}$ stage } \\
\hline N1 & & & & \multicolumn{3}{|c|}{ Ref } \\
\hline $\mathrm{N} 2$ & - & - & - & 2.314 & $1.077-4.971$ & $.032^{\mathrm{a}}$ \\
\hline N3 & - & - & - & 1.948 & $0.789-4.809$ & .148 \\
\hline \multicolumn{7}{|c|}{ Hormone receptor status } \\
\hline$\underline{E R+/ P R+}$ & \multicolumn{3}{|c|}{ Ref } & \multicolumn{3}{|c|}{ Ref } \\
\hline ER-/PR- & 2.100 & $0.790-5.583$ & .137 & 1.210 & $0.589-2.487$ & .604 \\
\hline
\end{tabular}

Abbreviations: DCH, docetaxel/cyclophosphamide; ER, estrogen receptor; HR, hazard ratio; LVI, lymphovascular invasion; NA, not applicable; PR, progesterone receptor; $\mathrm{TCH}$, docetaxel/carboplatin/trastuzumab; U-CCl, updated Charlson comorbidity index. ${ }^{a} P<.05$.

subgroup analysis of ShortHER by nodal status, noninferiority cutoffs were met for node-negative tumors. Moreover, with the favorable toxicity profile of trastuzumab and universal funding within the Canadian healthcare system, most oncologists are diligent in the administration of a complete course.

Another limitation of our study was the lack of prospective toxicity data. In our node-positive cohort, use of an anthracycline should ideally be weighed against longer-term cardiac morbidity. However, $<2 \%$ of patients in the node-positive cohort had a preexisting cardiac arrhythmia or history of congestive heart failure at baseline, as assessed by the treating radiation or medical oncologist's consultation reports (data not shown). Further limiting patient comorbidity as a factor in survival analyses is that approximately $98 \%$ of patients had a score of 0 to 1 on the U-CCI, indicative of a relatively healthy population.

\section{Conclusions}

Overall, this study provides valuable information with respect to the management of HER2+ breast cancer by further substantiating de-escalation of chemotherapy in the node-negative population while validating the effectiveness of FEC-DH in the node-positive population.

Submitted March 8, 2018; accepted for publication July 30, 2018.

Disclosures: The authors have disclosed that they have not received any financial considerations from any person or organization to support the preparation, analysis, results, or discussion of this article.

Author contributions: Study concept: Veitch, King, Tang, Lupichuk. Data acquisition: Veitch, Khan. Oversight and coordination of data management: Kostaras. Data cleaning: Veitch, Tilley. Data analysis: Tilley, Kostaras. Data interpretation: Veitch, Tilley, Ribnikar, Kostaras, King, Tang, Lupichuk. Manuscript preparation: Veitch, Kostaras. Manuscript editing: Khan, Tilley, Ribnikar, King, Tang, Lupichuk. Final approval: Veitch, Lupichuk.

Correspondence: Sasha Lupichuk, MD, MSc, Department of Oncology, Tom Baker Cancer Centre, 1331 29th Street NW, Calgary, AB Canada T2N4N2.

Email: Sasha.Lupichuk@AHS.ca 


\section{References}

1. Slamon DJ, Clark GM, Wong SG, et al. Human breast cancer: correlation of relapse and survival with amplification of the HER-2/neu oncogene. Science 1987;235:177-182.

2. Seshadri R, Firgaira FA, Horsfall DJ, et al. Clinical significance of HER-2/ neu oncogene amplification in primary breast cancer. The South Australian Breast Cancer Study Group. J Clin Oncol 1993;11:1936-1942.

3. Cameron D, Piccart-Gebhart MJ, Gelber RD, et al. 11 years' follow-up of trastuzumab after adjuvant chemotherapy in HER2-positive early breast cancer: final analysis of the HERceptin Adjuvant (HERA) trial. Lancet 2017:389:1195-1205.

4. Perez EA, Romond EH, Suman VJ, et al. Trastuzumab plus adjuvant chemotherapy for human epidermal growth factor receptor 2-positive breast cancer: planned joint analysis of overall survival from NSABP B-31 and NCCTG N9831. J Clin Oncol 2014;32:3744-3752.

5. Slamon D, Eiermann W, Robert N, et al. Adjuvant trastuzumab in HER2-positive breast cancer. N Engl J Med 2011;365:1273-1283.

6. Campiglio M, Bufalino R, Sasso M, et al. Effect of adjuvant trastuzumab treatment in conventional clinical setting: an observational retrospective multicenter Italian study. Breast Cancer Res Treat 2013;141:101-110.

7. Vici P, Pizzuti L, Natoli $C$, et al. Outcomes of HER2-positive early breast cancer patients in the pre-trastuzumab and trastuzumab eras: a real-world multicenter observational analysis. The RETROHER study. Breast Cancer Res Treat 2014;147:599-607.

8. Chia S, Norris B, Speers C, et al. Human epidermal growth factor receptor 2 overexpression as a prognostic factor in a large tissue microarray series of node-negative breast cancers. J Clin Oncol 2008;26:56975704 .

9. O'Sullivan CC, Bradbury I, Campbell C, et al. Efficacy of adjuvant trastuzumab forpatients with human epidermal growth factor receptor 2 -positive early breast cancer and tumors $\leq 2 \mathrm{~cm}$ : a meta-analysis of the randomized trastuzumab trials. J Clin Oncol 2015;33:2600-2608.

10. van Ramshorst MS, van der Heiden-van der Loo M, Dackus GM, et al. The effect of trastuzumab-based chemotherapy in small node-negative HER2-positive breast cancer. Breast Cancer Res Treat 2016;158:361371.

11. Curigliano G, Viale G, Bagnardi V, et al. Clinical relevance of HER2overexpression/amplification in patients with small tumor size and node-negative breast cancer. J Clin Oncol 2009;27:5693-5699.

12. Sparano JA, Zhao F, Martino S, et al. Long-term follow-up of the E1199 phase III trial evaluating the role of taxane and schedule in operable breast cancer. J Clin Oncol 2015;33:2353-2360.

13. Jones SE, Collea R, Paul D, et al. Adjuvant docetaxel and cyclophosphamide plus trastuzumab in patients with HER2-amplified early stage breast cancer: a single-group, open-label, phase 2 study. Lancet Oncol 2013;14:1121-1128.

14. Tolaney SM, Barry WT, Dang CT, et al. Adjuvant paclitaxel and trastuzumab for node-negative, HER2-positive breast cancer. N Engl J Med 2015;372:134-141.

15. Slamon D, Eiermann W, Robert N. Ten-year follow-up of BCIRG-006 comparing doxorubicin plus cyclophosphamide followed by docetaxel with doxorubicin plus cyclophosphamide followed by docetaxel and trastuzumab with docetaxel, carboplatin and trastuzumab in HER2-positive early breast cancer patients [abstract]. Presented at the 2015 San Antonio Breast Cancer Symposium; December 8-12, 2015; San Antonio, Texas. Abstract S5-04

16. Denduluri N, Somerfield MR, Eisen A, et al. Selection of optimal adju- vant chemotherapy regimens for human epidermal growth factor receptor 2 (HER2)-negative and adjuvant targeted therapy for HER2-positive breast cancers: an American Society of Clinical Oncology guideline adaptation of the Cancer Care Ontario Clinical Practice Guideline. J Clin Oncol 2016;34:2416-2427.

17. Quan H, Li B, Couris CM, et al. Updating and validating the Charlson comorbidity index and score for risk adjustment in hospital discharge abstracts using data from 6 countries. Am J Epidemiol 2011;173:676682.

18. Joensuu H, Bono P, Kataja $V$, et al. Fluorouracil, epirubicin, and cyclophosphamide with either docetaxel or vinorelbine, with or without trastuzumab, as adjuvant treatments of breast cancer: final results of the FinHer trial. J Clin Oncol 2009;27:5685-5692.

19. Lee HJ, Park IA, Park SY, et al. Two histopathologically different diseases: hormone receptor-positive and hormone receptor-negative tumors in HER2-positive breast cancer. Breast Cancer Res Treat 2014;145:615623.

20. Vici P, Pizzuti L, Natoli $C$, et al. Triple positive breast cancer: a distinct subtype? Cancer Treat Rev 2015;41:69-76.

21. Di Leo A, Desmedt C, Bartlett JM, et al. HER2 and TOP2A as predictive markers for anthracycline-containing chemotherapy regimens as adjuvant treatment of breast cancer: a meta-analysis of individual patient data. Lancet Oncol 2011;12:1134-1142.

22. Loi S, Dafni U, Karlis D, et al. Effects of estrogen receptor and human epidermal growth factor receptor-2 levels on the efficacy of trastuzumab: a secondary analysis of the HERA trial. JAMA Oncol 2016;2:10401047.

23. Schneeweiss A, Chia S, Hickish T, et al. Pertuzumab plus trastuzumab in combination with standard neoadjuvant anthracycline-containing and anthracycline-free chemotherapy regimens in patients with HER2-positive early breast cancer: a randomized phase II cardiac safety study (TRYPHAENA). Ann Oncol 2013;24:2278-2284.

24. Schneeweiss A, Chia S, Hickish T, et al. Long-term efficacy analysis of the randomised, phase II TRYPHAENA cardiac safety study: evaluating pertuzumab and trastuzumab plus standard neoadjuvant anthracycline-containing and anthracycline-free chemotherapy regimens in patients with HER2-positive early breast cancer. Eur J Cancer 2018;89:27-35.

25. Von Minckwitz G, Untch M, Blohmer JU, et al. Definition and impact of pathologic complete response on prognosis after neoadjuvant chemotherapy in various intrinsic breast cancer subtypes. J Clin Oncol 2012;30:1796-1804.

26. Von Minckwitz G, Procter M, de Azambuja E, et al. Adjuvant pertuzum$\mathrm{ab}$ and trastuzumab in early HER2-positive breast cancer. N Engl J Med 2017;377:122-131.

27. Martin M, Holmes FA, Ejlertsen $B$, et al. Neratinib after trastuzumab-based adjuvant therapy in HER2-positive breast cancer (ExteNET): 5-year analysis of a randomized, double-blind, placebo-controlled, phase 3 trial. Lancet Oncol 2017;18:1688-1700.

28. Gradishar WJ, Anderson BO, Abraham J, et al. NCCN Clinical Practice Guidelines in Oncology: Breast Cancer. Version 3.2018. Accessed December 2, 2018. To view the most recent version of these guidelines, visit NCCN.org.

29. Conte P, Frassoldati A, Bisagni G, et al. Nine weeks versus 1 year adjuvant trastuzumab in combination with chemotherapy: final results of the phase III randomized Short-HER study. Ann Oncol 2018;29:2328-2333. 